\title{
Successful percutaneous treatment with the Konar MFTM-VSD Occluder in an infant with Abernethy syndrome-case report
}

\author{
Petra Loureiro', Stanimir Georgiev ${ }^{2}$, Peter Ewert ${ }^{2}$, Daniel Tanase ${ }^{2}$, Andreas Eicken², Birgit Kammer ${ }^{3}$, \\ Robert Dalla-Pozza ${ }^{4}$ \\ ${ }^{1}$ Department of Pediatric Cardiology and Congenital Heart Disease, Hospital de Santa Marta, Nova University Lisbon, Lisbon, Portugal; \\ ${ }^{2}$ Department of Pediatric Cardiology and Congenital Heart Disease, German Heart Center Munich, Technische Universität München, Munich, \\ Germany; ${ }^{3}$ Department of Pediatric Radiology, ${ }^{4}$ Department of Pediatric Cardiology, Ludwig-Maximilians-Universität München, Munich, Germany \\ Correspondence to: Petra Loureiro, MD. Department of Pediatric Cardiology and Congenital Heart Disease, Hospital de Santa Marta - Centro \\ Hospitalar Lisboa Central-EPE, Lisboa, Rua de Santa Marta, 1169-024 Lisbon, Portugal. Email: petra.loureiro@gmail.com.
}

\begin{abstract}
Cyanosis persisting after surgical repair of complex congenital heart disease (CHD) may be related to the underlying disease. However, extracardiac causes should be also considered. We report on a patient with heterotaxy syndrome and double outlet right ventricle, in whom postoperative cyanosis was associated with an Abernethy malformation type II causing a hepatopulmonary syndrome. Despite this complex anatomy, interventional closure of the portosystemic shunt was done with a Konar MF ${ }^{\mathrm{TM}}-\mathrm{VSD}$ Occluder. The patient recovered rapidly with relief of cyanosis within one month. This case highlights the importance of a careful diagnostic assessment in patients with complex CHD, who presents cyanoses after surgical repair. In addition, it shows the feasibility and safety of a percutaneous approach with complete closure of the vascular malformation in a patient with a complex anatomy.
\end{abstract}

Keywords: Abernethy malformation; congenital heart disease (CHD); cyanosis; hepatopulmonary syndrome; case report

Submitted Mar 22, 2020. Accepted for publication Jun 12, 2020.

doi: $10.21037 / \mathrm{cdt}-20-380$

View this article at: http://dx.doi.org/10.21037/cdt-20-380

\section{Introduction}

Abernethy malformation of the portal vein results in diverting portal venous blood flow towards the inferior vena cava (IVC) bypassing the hepatic parenchyma $(1,2)$. Patients may develop hepatic failure, hyperammonemia, hepatic encephalopathy, or hepatopulmonary syndrome among other complications $(1,3)$. In hepatopulmonary syndrome, associated with pulmonary arteriovenous fistulae, cyanosis may be one of the main manifestations. In children with complex congenital heart disease (CHD), especially in those with malformations of the right heart and limited pulmonary blood flow, cyanosis is a frequent symptom. After corrective heart surgery, cyanosis is usually interpreted as a symptom of limited pulmonary blood flow improving slowly as pulmonary arteries are growing (4). We report on an infant with CHD in whom, after cardiac surgery, cyanosis was not related to the underlying disease. A thorough investigation led to the diagnosis of an Abernethy malformation type II with two variants of anatomical connections-an interrupted IVC and azygos continuity and a left renal vein connected to the dilated portal vein. To the best of our knowledge, this is the first report of an infant with CHD and an Abernethy malformation type II with two variants of anatomical connections, successfully treated percutaneously with a Konar MF $\mathrm{MF}^{\mathrm{TM}}$ VSD Occluder. This case report is written in accordance with Care Guideline (available at http://dx.doi. org/10.21037/cdt-20-380).

\section{Case presentation}

A 7-months old boy $(5.95 \mathrm{~kg}, 72 \mathrm{~cm})$ was admitted to the hospital, two months after corrective heart surgery, for low weight gain, tachydyspnea and progressing cyanosis [peripheral oxygen saturations $\left(\mathrm{SpO}_{2}\right)$ 83-87\%] (Tables 1,2). 
Table 1 Past medical history and interventions

\begin{tabular}{ll}
\hline Date & Past medical history and interventions \\
\hline 10. Feb. 2019 & $\begin{array}{l}\text { Birth. Diagnosis: heterotaxy syndrome, Fallot-type double outlet right ventricle, ventricular septal defect, atrial septal } \\
\text { defect and subvalvular and valvular pulmonary stenosis }\end{array}$ \\
22. Jul. 2019 & Cardiac surgery-anatomical correction \\
\hline
\end{tabular}

Table 2 Timeline of interventions and outcomes

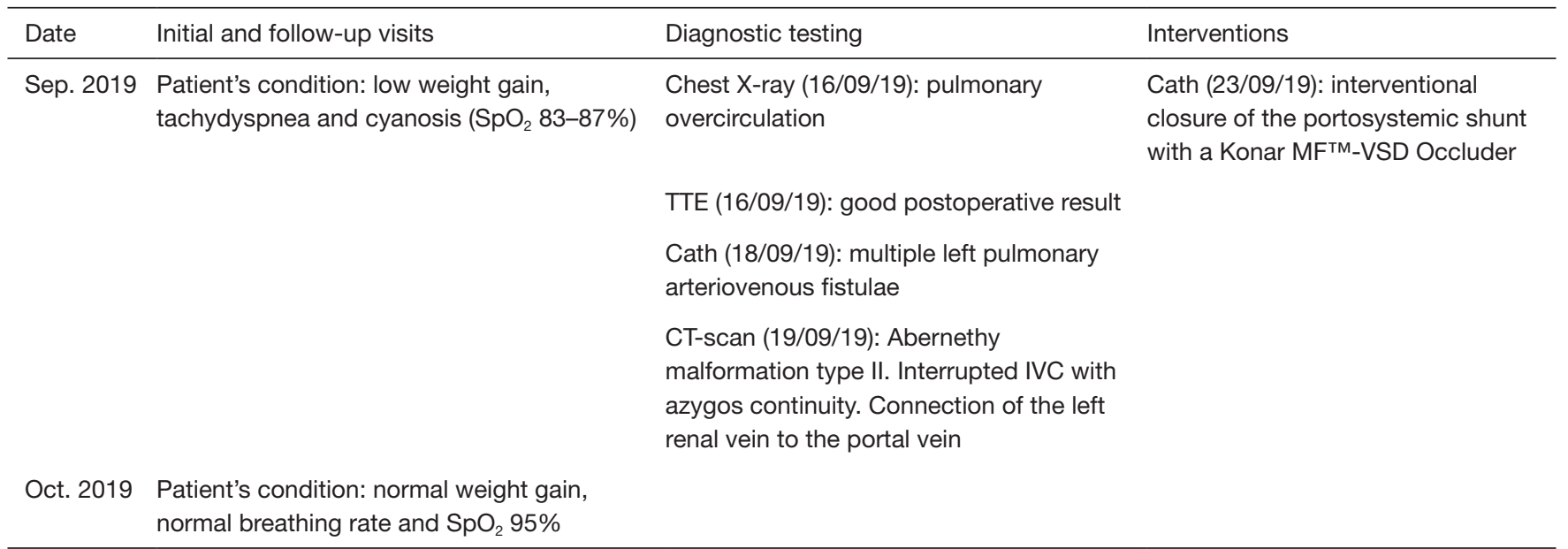

Final outcome: successful percutaneous treatment of an Abernethy malformation type II.

The patient was born with heterotaxy syndrome, Fallottype double outlet right ventricle, ventricular septal defect (VSD), atrial septal defect and subvalvular and valvular pulmonary stenosis. Anatomical correction was performed with 5 months of age. On the admission the $\mathrm{SpO}_{2}$ improved with oxygen supply and the chest $\mathrm{X}$-ray was suggestive for pulmonary overcirculation. Transthoracic echocardiogram showed good postoperative result without intracardiac right-to-left shunt explaining the cyanosis of the patient. A diagnostic cardiac catheterization was performed. Blood gas samples from the right pulmonary veins showed an oxygen saturation of $100 \%$. The left pulmonary veins were however significantly desaturated (70\% and $84 \%)$. Angiographies excluded intracardiac right-to-left-shunt and showed multiple left pulmonary arteriovenous fistulae with an atypical pulmonary vascular pattern, a diffusely dilated pulmonary vascular bed and accelerated pulmonary bloodpassage (Figure 1). A CT-scan showed thoracal bilateral leftsidedness, interrupted IVC with a prominent azygos vein draining in the superior vena cava, polysplenia and a bilobed liver connected to a dilated portal vein (diameter of $9 \mathrm{~mm}$ ) which, in its intrahepatic course, appeared to be hypoplastic (diameter $2 \mathrm{~mm}$ ). The diagnosis of an Abernethy malformation type II (hypoplastic portal vein with portal blood diversion into the IVC through a side-to-side extrahepatic communication) was made (Figure 2). However, in this patient two variants of anatomical connections were present, first as there was an interrupted IVC and azygos continuity, the dilated portal vein was connected to the azygos vein. Second, the left renal vein was connected to the dilated portal vein. As a consequence of the portosystemic shunt through the Abernethy malformation, the patient had developed a hepatopulmonary syndrome, resulting in intrapulmonary arteriovenous fistulae with right-to-left shunt leading to cyanosis and dyspnea.

A cardiac catheterization was scheduled to close the malformation. The patient's parents provided written informed consent for the procedure. $\mathrm{SpO}_{2}$ was $60 \%$ (Figure 3). From a transjugular approach, a diagnostic catheter was advanced into the azygos vein and the Abernethy vessel. Angiography confirmed the anatomy of a $10 \mathrm{~mm}$-wide tortuous portal vein. Test-balloon occlusion showed a portal vein pressure of $14 \mathrm{mmHg}$, and selective contrast injection during balloon occlusion revealed drainage of the splanchnic veins and of the splenic vena into the distal part of the vessel, whereas the left 


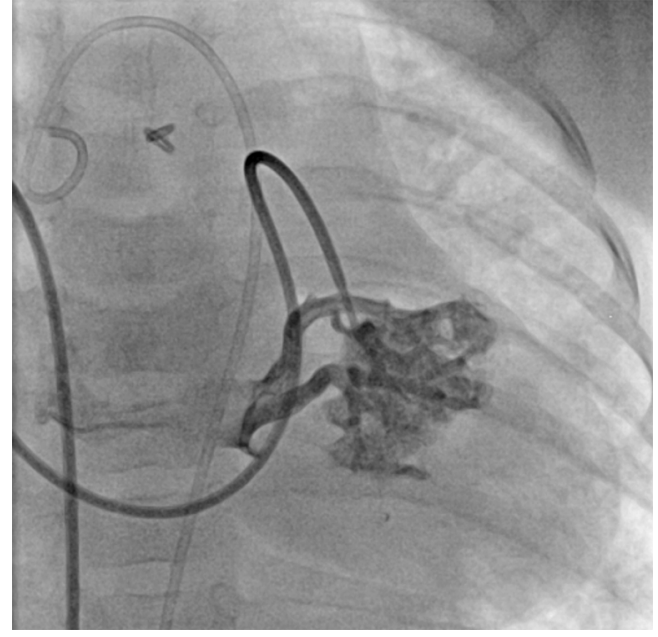

Figure 1 Angiography showing left pulmonary arteriovenous fistulae in a patient with a complex congenital heart disease after anatomical surgical correction.

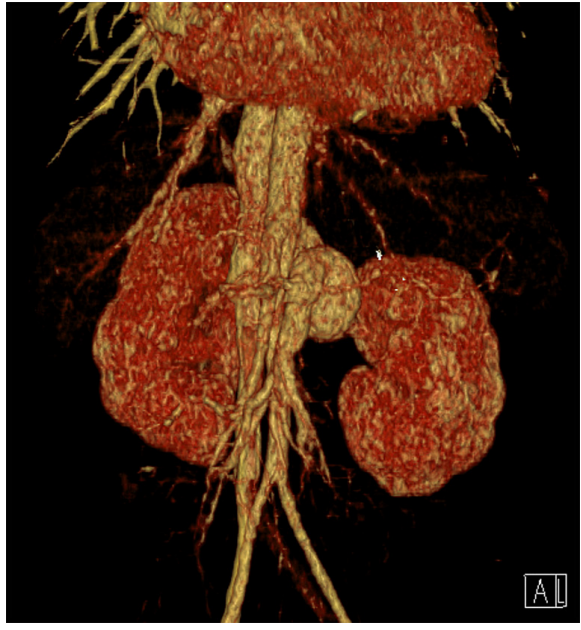

Figure 2 CT scan demonstrating an Abernethy malformation type II.
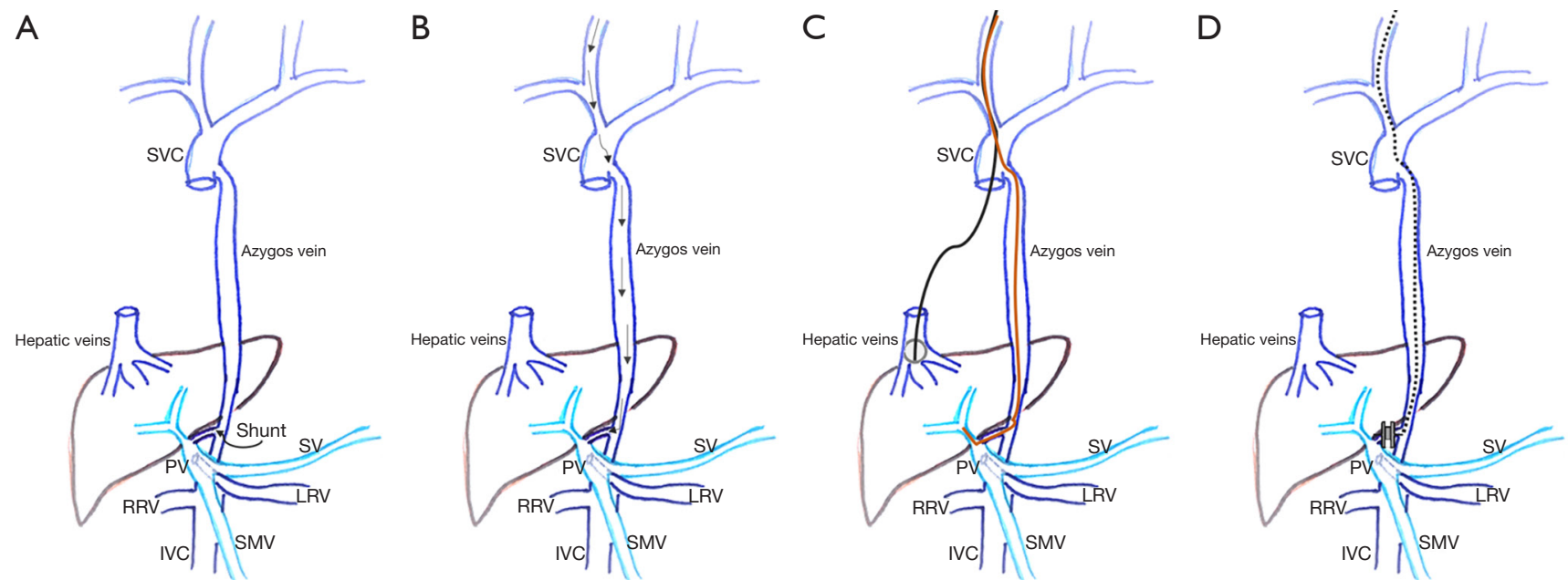

Figure 3 Cardiac catheterization schema. (A) Abernethy malformation type II - Shunt between portal vein and azygos vein. (B) Diagnostic cardiac catheterization - from the left internal jugular vein through the azygos vein until the shunt. (C) Wedge pressure measurement of the portal venous system (black line) and selective contrast injection during balloon occlusion (brown line). (D) interventional closure of the portosystemic shunt. IVC, inferior vena cava; LRV, left renal vein; PV, portal vein; RRV, right renal vein; SMV, superior mesenteric vein; SV, splenic vein; SVC, superior vena cava.

renal vein drained into the proximal part of the siphonlike portal vein close to its connection to the azygos vein. The intrahepatic segment of the portal vein opened nicely during selective injection (Figure 4). A 14/12 mm device designed for VSD-closure (Konar MFTM_VSD Occluder, Lifetech Scientific Inc., Shenzhen, China) was successfully implanted through a $7 \mathrm{~F}$ sheath between the connections, diverting the splanchnic venous blood flow towards the intrahepatic segments of the portal vein and preserving the renal vein connection (Figure 5). In our case, there were no complications during the procedure. However, regarding adverse/unanticipated events, one of the main ones is the embolization or malposition of the device. Therefore, in this case, we chose a device attached to a flexible delivery wire 


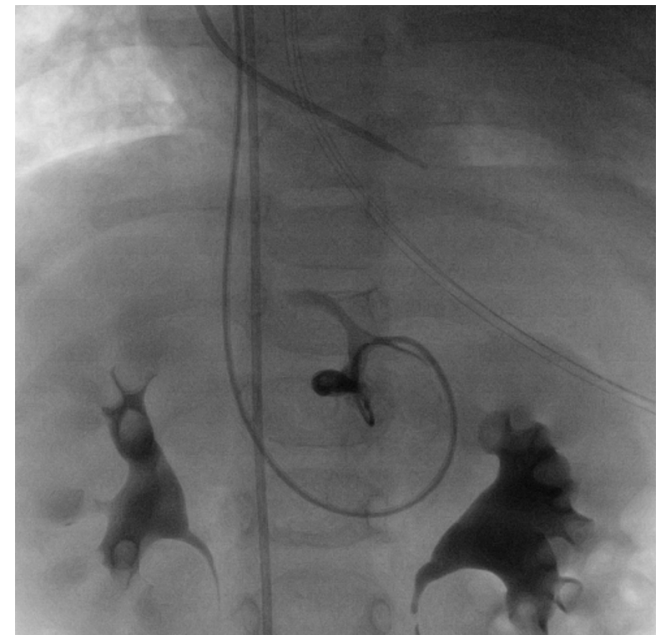

Figure 4 Selective angiography showing the hypoplastic intrahepatic segment of the portal vein.

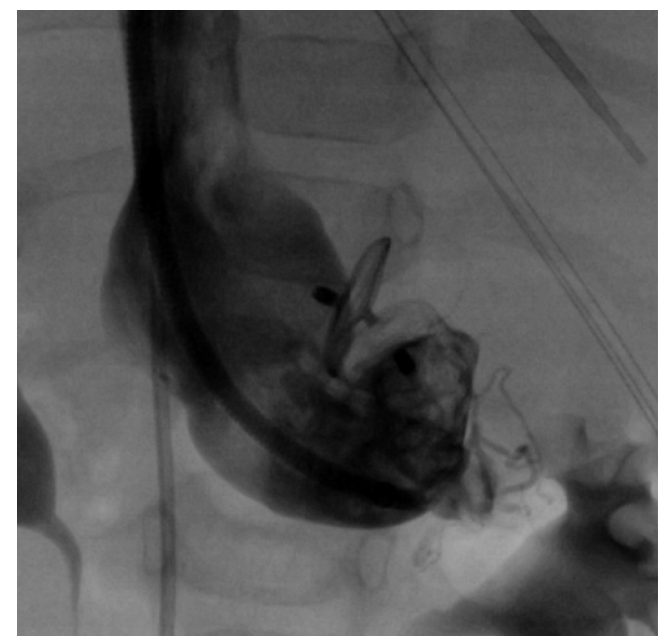

Figure 5 Interventional closure of the portosystemic shunt with a 14/12 mm Konar MF ${ }^{\mathrm{TM}}$-VSD Occluder.

which allowed a precise placement with minimal movement or distortion during the release. After the intervention, the patient showed rapid improvement with the $\mathrm{SpO}_{2}$ returning gradually to normal levels. One month after the procedure the $\mathrm{SpO}_{2}$ was $95 \%$. On the latest follow-up the patient was clinically and hemodynamically stable, presenting a good growth development and a $\mathrm{SpO}_{2}$ of $95 \%$.

\section{Discussion}

Abernethy malformation comprises rare malformations of the portal vein system with absent (Type I) or hypoplastic (Type II) intrahepatic portal veins (2) (Figure 6). So far, only few cases have been described (5). Hyperammonemia, hepatic failure and hepatic encephalopathy appear as a consequence of the bypass of the portal venous blood from the hepatic parenchyma (1). This malformation was also associated with hepatopulmonary syndrome, which defines patients with hepatic failure and progressive pulmonary cyanosis due to pulmonary vascular changes leading to diffuse arteriovenous fistulae (3). As the experience in patients with Abernethy malformation and hepatopulmonary syndrome is limited, treatment strategies must be tailored on a case-by-case basis (6). In general, patients with hepatopulmonary syndrome showed improvement of cyanosis when the hepatic function was restored or the portosystemic shunt was abolished (7). Adult patients with small shunts are amenable to interventional procedures such as device closure (8), while small children are usually selected for surgical ligation of the portosystemic shunt or orthotopic liver transplantation $(7,9)$. Only occasional cases of transcatheter closure of the shunting vessel at a pediatric age were reported (10).

Our patient presented a large portosystemic shunt to the azygos vein and an anatomic variant of a left renal vein connected to the portal vein. In this case, the clinical condition of the patient showed a rapidly worsening, so a timely closure of the Abernethy malformation was mandatory. A percutaneous approach was chosen, although the feasibility of interventional closure could be doubtful, as that the occluder had to be placed exactly between the connections of the splanchnic and the renal veins. A transjugular access was required as there was continuity of the azygos vein and therefore the portal vein had to be entered in a cranio-caudal direction. As mentioned in previous publications, a wedge pressure recording of the portal venous system over a certain period of time is mandatory in a hypoplastic intrahepatic portal vein system (8). In this case, during balloon occlusion the portal vein pressure remained unchanged at $14 \mathrm{mmHg}$. A Konar $M^{T M}$-VSD Occluder was chosen for the procedure. This device is relatively short, has a small rim, a waist length of $4 \mathrm{~mm}$ and is attached to a flexible delivery wire allowing a precise placement without distortion during release. Following the intervention, the patient recovered rapidly with relief of cyanosis within one month.

In patients with Abernethy malformation and hepatopulmonary syndrome, the therapeutic options 


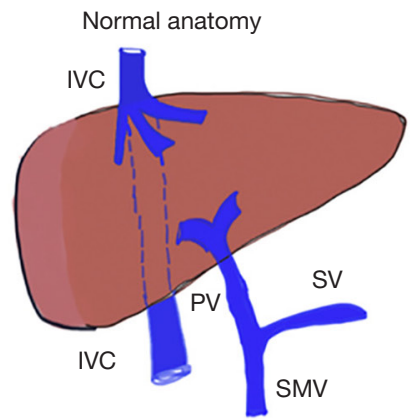

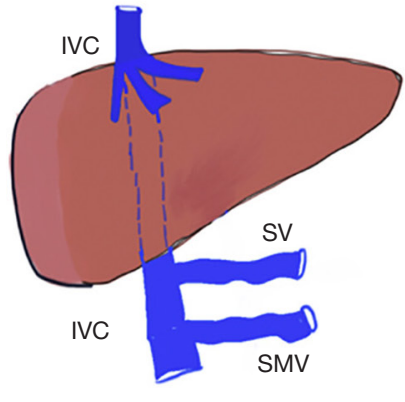

Type 1a

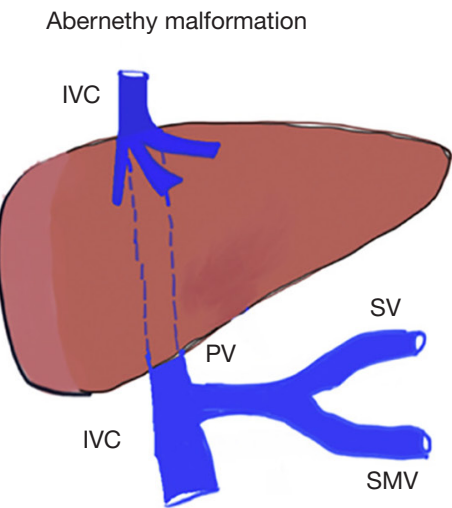

Type 1b

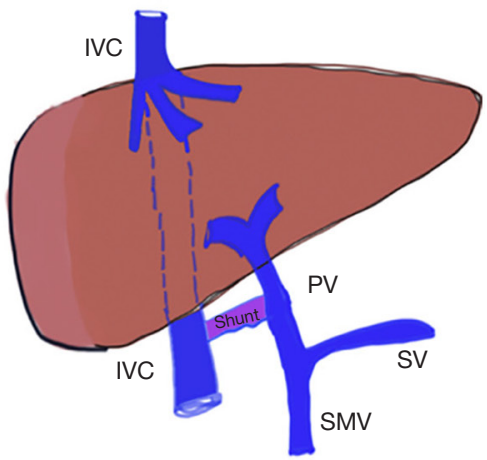

Type 2

Figure 6 Normal anatomy: Normal portovenous anatomy. Type 1a: Splenic vein (SV) and superior mesenteric vein (SMV) drain directly into the inferior vena cava (IVC). Type 1b: Portal vein (PV) drains directly into the IVC. Type 2: Shunt between PV and IVC. Reprinted from "Abernethy malformation type 2: varied presentation, management and outcome”, by Jain V, Sangdup T, Agarwala S, et al. J Pediatr Surg, 2019;54(4):760-765. Copyright 2018 by Elsevier Inc. Reprinted with permission.

should consider the underlying anatomy. In Abernethy malformation type I orthotopic liver transplantation is the therapy of choice, while in Abernethy malformation type II surgical shunt ligation is usually performed. In a literature review, Osorio et al. (6) reported six patients with Abernethy malformation and hepatopulmonary syndrome, four of whom underwent surgical therapy (two orthotopic liver transplantation, two surgical shunt ligation). Knirsch et al. (10) reports on a series of eight patients with portosystemic shunts, two of whom had a hepatopulmonary syndrome. Both patients were interventionally treated with occlusion of the malformation. Nevertheless, cyanosis after therapy may persist for months or even years.

Thought this case, we emphasize the importance of a careful diagnostic evaluation of a cyanotic infant with corrected complex CHD. We also demonstrated the feasibility of the percutaneous treatment and successful closure of the malformation in an infant with an Abernethy malformation type II with variants of anatomical connections.

\section{Strengths and limitations}

The diagnosis of a rare pathology, as Abernethy malformation, in a patient with a corrected complex CHD can be delayed, as cyanosis is a common manifestation and is usually associated with residual lesions. However, the discussion and approach of these patients by a multidisciplinary team allows a timely diagnosis and treatment. Transcatheter closure of the shunting vessel at a pediatric age is a challenging procedure, requiring an experienced team and adequate material.

\section{Conclusions}

We report an atypical Abernethy malformation and hepatopulmonary syndrome type II in a cyanotic infant with corrected complex CHD. Despite the anatomic challenges due to an interrupted IVC, a left renal vein connected to the malformation and a severe hypoplastic intrahepatic portal 
venous segment, transcatheter occlusion of the malformation was feasible and led to a rapid recovery of the patient.

\section{Acknowledgments}

Funding: None.

\section{Footnote}

Reporting Checklist: The authors have completed the CARE reporting checklist. Available at http://dx.doi. org/10.21037/ cdt-20-380

Peer Review File: Available at http://dx.doi. org/10.21037/ cdt-20-380

Provenance and Peer Review: This article was commissioned by the Guest Editors (Yskert von Kodolitsch, Harald Kaemmerer, Koichiro Niwa) for the series "Current Management Aspects in Adult Congenital Heart Disease (ACHD): Part III" published in Cardiovascular Diagnosis and Therapy. The article has undergone external peer review.

Conflicts of Interest: All authors have completed the ICMJE uniform disclosure form (available at http:// dx.doi.org/10.21037/cdt-20-380). The series "Current Management Aspects in Adult Congenital Heart Disease (ACHD): Part III" was commissioned by the editorial office without any funding or sponsorship. The authors have no other conflicts of interest to declare.

Ethical Statement: The authors are accountable for all aspects of the work in ensuring that questions related to the accuracy or integrity of any part of the work are appropriately investigated and resolved. All procedures performed in studies involving human participants were in accordance with the ethical standards of the institutional and/or national research committee(s) and with the Helsinki Declaration (as revised in 2013). Written informed consent was obtained from the patient for publication of this manuscript and any accompanying images.

Open Access Statement: This is an Open Access article distributed in accordance with the Creative Commons Attribution-NonCommercial-NoDerivs 4.0 International License (CC BY-NC-ND 4.0), which permits the noncommercial replication and distribution of the article with the strict proviso that no changes or edits are made and the original work is properly cited (including links to both the formal publication through the relevant DOI and the license). See: https://creativecommons.org/licenses/by-nc-nd/4.0/.

\section{References}

1. Avila LF, Luis AL, Encinas JL, et al. [Congenital portosystemic shunt. The Abernethy malformation]. Cir Pediatr 2006;19:204-9.

2. Howard ER, Davenport M. Congenital extrahepatic portocaval shunts--the Abernethy malformation. J Pediatr Surg 1997;32:494-7.

3. Alvarez AE, Ribeiro AF, Hessel G, et al. Abernethy malformation: one of the etiologies of hepatopulmonary syndrome. Pediatr Pulmonol 2002;34:391-4.

4. Gedikbasi A, Oztarhan K, Gul A, et al. Diagnosis and prognosis in double-outlet right ventricle. Am J Perinatol 2008;25:427-34.

5. Witjes CD, Ijzermans JN, Vonk Noordegraaf A, et al. Management strategy after diagnosis of Abernethy malformation: a case report. J Med Case Rep 2012;6:167.

6. Osorio MJ, Bonow A, Bond GJ, et al. Abernethy malformation complicated by hepatopulmonary syndrome and a liver mass successfully treated by liver transplantation. Pediatr Transplant 2011;15:E149-51.

7. Raghuram KA, Bijulal S, Krishnamoorthy KM, et al. Regression of pulmonary vascular disease after therapy of Abernethy malformation in visceral heterotaxy. Pediatr Cardiol 2013;34:1882-5.

8. Venkateshwaran S, Krishnamoorthy KM, Sivasankaran S. Percutaneous device closure of Abernethy malformation -a treatable cause of hepatopulmonary syndrome. Catheter Cardiovasc Interv 2014;83:968-70.

9. Yilmaz C, Onen Z, Farajov R, et al. Live donor liver transplantation for a child presented with severe hepatopulmonary syndrome and nodular liver lesions due to Abernethy malformation. Pediatr Transplant 2017. doi: 10.1111/petr.12874.

10. Knirsch W, Benz DC, Bühr P, et al. Catheter interventional treatment of congenital portosystemic venous shunts in childhood. Catheter Cardiovasc Interv 2016;87:1281-92.

Cite this article as: Loureiro P, Georgiev S, Ewert P, Tanase D, Eicken A, Kammer B, Dalla-Pozza R. Successful percutaneous treatment with the Konar MF ${ }^{\mathrm{TM}}$-VSD Occluder in an infant with Abernethy syndrome-case report. Cardiovasc Diagn Ther 2021;11(2):631-636. doi: 10.21037/cdt-20-380 